\title{
ALMA OBSERVATIONS OF THE HOST GALAXY OF GRB 090423 AT $z=8.23$ : DEEP LIMITS ON OBSCURED STAR FORMATION 630 MILLION YEARS AFTER THE BIG BANG
}

\author{
E. BERger ${ }^{1}$, B. A. ZaUderer ${ }^{1}$, R.-R. Chary ${ }^{1,2}$, T. LASKar ${ }^{1}$, R. CHORNOCK ${ }^{1}$, \\ N. R. Tanvir ${ }^{3}$, E. R. Stanway ${ }^{4}$, A. J. Levan ${ }^{4}$, E. M. LevesQue ${ }^{5}$, and J. E. Davies ${ }^{1}$ \\ ${ }^{1}$ Harvard-Smithsonian Center for Astrophysics, 60 Garden Street, Cambridge, MA 02138, USA \\ 2 Spitzer Science Center, California Institute of Technology, MC 220-6, 1200 East California Boulevard, Pasadena, CA 91125, USA \\ ${ }^{3}$ Department of Physics and Astronomy, University of Leicester, University Road, Leicester LE1 7RH, UK \\ ${ }^{4}$ Department of Physics, University of Warwick, Gibbet Hill Road, Coventry CV4 7AL, UK \\ ${ }^{5}$ CASA, University of Colorado UCB 389, Boulder, CO 80309, USA \\ Received 2014 August 11; accepted 2014 September 28; published 2014 November 12
}

\begin{abstract}
We present rest-frame far-infrared (FIR) and optical observations of the host galaxy of GRB 090423 at $z=8.23$ from the Atacama Large Millimeter Array (ALMA) and the Spitzer Space Telescope, respectively. The host remains undetected to $3 \sigma$ limits of $F_{v}(222 \mathrm{GHz}) \lesssim 33 \mu \mathrm{Jy}$ and $F_{\nu}(3.6 \mu \mathrm{m}) \lesssim 81 \mathrm{nJy}$. The FIR limit is about 20 times fainter than the luminosity of the local ULIRG Arp 220 and comparable to the local starburst M 82. Comparing this with model spectral energy distributions, we place a limit on the infrared (IR) luminosity of $L_{\mathrm{IR}}(8-1000 \mu \mathrm{m}) \lesssim 3 \times 10^{10} L_{\odot}$, corresponding to a limit on the obscured star formation rate of SFR $\mathrm{IR}_{\mathrm{IR}} \lesssim M_{\odot} \mathrm{yr}^{-1}$. For comparison, the limit on the unobscured star formation rate from Hubble Space Telescope rest-frame ultraviolet (UV) observations is $\mathrm{SFR}_{\mathrm{UV}} \lesssim 1 M_{\odot} \mathrm{yr}^{-1}$. We also place a limit on the host galaxy stellar mass of $M_{*} \lesssim 5 \times 10^{7} M_{\odot}$ (for a stellar population age of $100 \mathrm{Myr}$ and constant star formation rate). Finally, we compare our millimeter observations to those of field galaxies at $z \gtrsim 4$ (Lyman break galaxies, Ly $\alpha$ emitters, and submillimeter galaxies) and find that our limit on the FIR luminosity is the most constraining to date, although the field galaxies have much larger rest-frame UV/optical luminosities than the host of GRB 090423 by virtue of their selection techniques. We conclude that GRB host galaxies at $z \gtrsim 4$, especially those with measured interstellar medium metallicities from afterglow spectroscopy, are an attractive sample for future ALMA studies of high redshift obscured star formation.
\end{abstract}

Key words: galaxies: high-redshift - gamma-ray burst: individual (GRB 090423) - radio continuum: galaxies

Online-only material: color figures

\section{INTRODUCTION}

Thanks to their extreme brightness, gamma-ray bursts (GRBs) can be utilized as powerful probes of star and galaxy formation at high redshift, including during the epoch of reionization (e.g., Totani et al. 2006; Tanvir et al. 2009, 2012; Chornock et al. 2013, 2014). In particular, spectroscopy of the optical/ near-infrared (IR) afterglow emission can provide an unambiguous and precise spectroscopic redshift as well as a measurement of the host galaxy interstellar medium (ISM) properties independent of the host galaxy brightness (e.g., Totani et al. 2006; Chornock et al. 2013). This unique insight can then be combined with multi-wavelength photometric follow-up of the host galaxies to study properties such as their stellar mass and star formation activity (e.g., Laskaret al. 2011; Tanvir et al. 2012).

In contrast, direct galaxy studies at these redshifts rely either on photometric redshifts (the Ly $\alpha$ break technique; LBGs) and are therefore limited to ultraviolet (UV)-bright star forming galaxies, usually with no subsequent spectroscopic confirmation (e.g., Stanwayet al. 2003; Bunker et al. 2004; Malhotra et al. 2005; Bouwens et al. 2006, 2011; Ouchi et al. 2009; McLure et al. 2011; Vanzella et al. 2011; Ono et al. 2012; Finkelstein et al. 2013), or on narrow-band Ly $\alpha$ imaging (Ly $\alpha$ emitters; LAEs), which also requires significant star formation and subsequent spectroscopic confirmation (e.g., Kodaira et al. 2003; Iye et al. 2006; Hu et al. 2010; Ouchi et al. 2010; Shibuya et al. 2012). In the case of LAEs, the reliance on Ly $\alpha$ emission may also inhibit detections during the epoch of reionization due to significant scattering and absorption of $\operatorname{Ly} \alpha$ photons by the neutral intergalactic medium; the same effect also inhibits the success of LBG spectroscopic confirmation, which also relies on detection of the $\operatorname{Ly} \alpha$ line. Similarly, the reliance on restframe UV and Ly $\alpha$ emission bias both the LBG and LAE selection against dusty environments. Moreover, for both LBGs and LAEs, measurements of the ISM properties are beyond the reach of current instrumentation since the relevant restframe optical emission lines are redshifted into the mid-IR. An alternative technique of submillimeter color selection has also led to the identification of a few high-redshift submillimeterbright galaxies (SMGs), with spectroscopic confirmation via $\mathrm{CO}$ and [C $\mathrm{II}]$ emission lines (e.g., Walter et al. 2012; Riechers et al. 2013). However, these extremely luminous and highly starforming galaxies are rare and do not shed light on the bulk of the galaxy population. Thus, spectroscopically confirmed GRB host galaxies at high redshift provide an attractive sample for multi-wavelength follow-up observations.

In this context, the advent of the Atacama Large Millimeter Array (ALMA) makes it possible for the first time to probe obscured star formation in high-redshift galaxies to a sensitivity level of only a few $M_{\odot} \mathrm{yr}^{-1}$ or an integrated IR luminosity of $L_{\mathrm{IR}} \sim 10^{10} L_{\odot}$. Previously, only rare SMGs with $L_{\mathrm{IR}} \gtrsim 10^{12} L_{\odot}$ (ultra-luminous infrared galaxies; ULIRGs) and correspondingly high star formation rates of $\sim 10^{3} M_{\odot} \mathrm{yr}^{-1}$ were accessible (e.g., Cox et al. 2011; Combes et al. 2012; Walter et al. 2012; Riechers et al. 2013). In the long term, ALMA observations of GRB host galaxies with measured ISM metallicities will shed light on the relations between metallicity, dust content, and obscured star formation at redshifts that are inaccessible with other techniques (i.e., $z \gtrsim 4$ ). This will also provide a 
Table 1

Observations of the Host Galaxy of GRB 090423

\begin{tabular}{lcccc}
\hline \hline Instrument & $\lambda_{\text {obs }}$ & $\begin{array}{c}F_{v}^{\mathrm{a}} \\
(\mu \mathrm{Jy})\end{array}$ & $\begin{array}{c}L_{v}^{\mathrm{a}} \\
\left(\mathrm{erg} \mathrm{s}^{-1} \mathrm{~Hz}^{-1}\right)\end{array}$ & Reference \\
\hline ATCA & $0.79 \mathrm{~cm}$ & $\lesssim 9.3$ & $\lesssim 8.7 \times 10^{30}$ & Stanway et al. (2011) \\
ALMA & $0.14 \mathrm{~cm}$ & $\lesssim 33$ & $\lesssim 3.1 \times 10^{31}$ & This paper \\
Spitzer/IRAC & $3.6 \mu \mathrm{m}$ & $\lesssim 8.1 \times 10^{-2}$ & $\lesssim 7.6 \times 10^{28}$ & This paper \\
HST/WFC3 & $1.54 \mu \mathrm{m}$ & $\lesssim 9.0 \times 10^{-3}$ & $\lesssim 8.6 \times 10^{27}$ & Tanvir et al. (2012) \\
HST/WFC3 & $1.25 \mu \mathrm{m}$ & $\lesssim 6.0 \times 10^{-3}$ & $\lesssim 5.6 \times 10^{27}$ & Tanvir et al. (2012) \\
\hline
\end{tabular}

Note. ${ }^{\text {a }}$ Limits are $3 \sigma$.

comparison with the rest-frame FIR properties of LBGs and LAEs, although none have been individually detected to date at $z \gtrsim 4$ (e.g., Ouchi et al. 2013; Ota et al. 2014).

Here, we present deep ALMA observations of the host galaxy of the most distant spectroscopically confirmed burst to date-GRB 090423 at $z=8.23$ (Salvaterra et al. 2009; Tanvir et al. 2009). The observations are aimed at reaching a restframe FIR luminosity level below that of a luminous infrared galaxy (LIRG; $L_{\mathrm{IR}}=10^{11} L_{\odot}$ ). We also present Spitzer Space Telescope rest-frame optical observations to constrain the stellar mass of the host galaxy. The ALMA and Spitzer observations and data analysis are described in Section 2. In Section 3, we use the data to constrain the host galaxy properties and in Section 4, we compare these results to millimeter studies of LAEs, LBGs, and SMGs at $z \gtrsim 4$.

\section{OBSERVATIONS}

The discovery and redshift determination of GRB 090423 are presented in Tanvir et al. (2009), who find $z=8.23_{-0.07}^{+0.06}$. The position of the afterglow is R.A. $=09^{\mathrm{h}} 55^{\mathrm{m}} 33^{\mathrm{s}} .29$, decl. $=+18^{\circ} 08^{\prime} 57^{\prime \prime} .8$ (J2000) with an uncertainty of about 0 . $^{\prime \prime} 1$ in each coordinate. The location of GRB 090423 was previously observed with the Hubble Space Telescope Wide-field Camera 3 (HST/WFC3) in the F110W and F160W filters, leading to non-detections (Tanvir et al. 2012). It was also observed with the Australia Telescope Compact Array (ATCA), yielding non-detections of rest-frame $850 \mu \mathrm{m}$ continuum emission and CO (3-2) emission (Stanway et al. 2011). The continuum limits are summarized in Table 1.

\subsection{Atacama Large Millimeter Array}

ALMA band $6(222 \mathrm{GHz})$ observations were carried out with 25-28 $12 \mathrm{~m}$ diameter antennas in five observing blocks spread over 12 days on 2013 November 18, 21, and 30 UT (Cycle 1). The $2 \mathrm{GHz}$ wide spectral windows were set to central frequencies of $213,215,229$, and $231 \mathrm{GHz}$. The total on-source integration time was $163 \mathrm{~min}$ after data flagging. We performed data calibration and imaging using the Common Astronomy Software Application. The gain calibration utilized J 0854+2006, while bandpass calibration utilized one of $\mathrm{J}$ 0522-3627, J 0538-4405, J 1037-2934, or J 1058+0133 in each observing block. Absolute flux calibration was performed using observations of the solar system objects Ceres, Ganymede, or Pallas. We found some minor variations in the resulting fluxes of J 0854+2006 between the observing blocks, likely due to the use of asteroids, which have uncertain flux models. We therefore used Ganymede when possible and Ceres to determine and manually set the flux for $\mathrm{J} 0854+2006$ in each epoch. ${ }^{6}$ The overall uncertainty in the absolute flux calibration is about $10 \%$.

The resulting combined continuum map (natural weighting without primary beam correction) has an rms noise of $11 \mu \mathrm{Jy}$ beam $^{-1}$ for a synthesized beam size (FWHM) of $1^{\prime \prime} .0 \times$ 0 '.80 (Figure 1). No source is detected at the location of GRB $090423^{7}$ (with a flux density at the source location of $-4 \pm 11 \mu \mathrm{Jy})$, leading an upper limit of $F_{\nu}(222 \mathrm{GHz}) \lesssim 33 \mu \mathrm{Jy}$ $(3 \sigma$; Table 1$)$.

\subsection{Spitzer Space Telescope}

Spitzer observations were obtained during the warm mission (DDT Program 538) with the Infrared Array Camera (IRAC) at $3.6 \mu \mathrm{m}$ on 2010 January 26 and 27 UT. Each observation consisted of $650100 \mathrm{~s}$ frames (93.6s on source), for a combined on-source time of $33.8 \mathrm{hr}$. We analyzed the data using the Great Observatories Origins Deep Survey pipeline, including corrections for bright star image artifacts (muxbleed and pulldown) with dark and sky background subtraction. We carried out astrometric alignment utilizing stars in common with the Sloan Digital Sky Survey database and created a combined mosaic of the individual frames on a $0.4 \times 0$.'4 pixel grid. We also produced a pair of split images, each with half of the frames, and used the difference in these split images to produce a noise map. The resulting uncertainty is about two times higher than that derived from pure pixel statistics in the vicinity of the source.

We do not detect significant emission at the location of GRB 090423 (Figure 1). There is no evidence for significant source confusion at this location, in agreement with the HST/ WFC3 images of the field (Tanvir et al. 2012). From the noise maps, we find that the $1 \sigma$ point source sensitivity at the location of GRB 090423 is $27 \mathrm{nJy}$. Photometry in a $1^{\prime \prime} .2$ radius aperture yields a $3.6 \mu \mathrm{m}$ flux density of $26 \pm 27 \mathrm{nJy}$, after aperture corrections. We therefore place an upper limit on the brightness of the host of $F_{\nu}(3.6 \mu \mathrm{m} \lesssim 81)$ nJy $(3 \sigma$; Table 1$)$.

\section{HOST GALAXY PROPERTIES: IR LUMINOSITY, OBSCURED STAR FORMATION RATE, AND STELLAR MASS}

The host galaxy of GRB 090423 remains undetected in our ALMA and Spitzer observations. The flux density limits are plotted in the left panel of Figure 2. Converted to restframe spectral luminosity, the non-detections correspond to $L_{v}(145 \mu \mathrm{m}) \lesssim 3.1 \times 10^{31} \mathrm{erg} \mathrm{s}^{-1} \mathrm{~Hz}^{-1}$ and $L_{v}(0.39 \mu \mathrm{m}) \lesssim$ $7.6 \times 10^{28} \mathrm{erg} \mathrm{s}^{-1} \mathrm{~Hz}^{-1}$, respectively (right panel of Figure 2 and Table 1).

Also shown in Figure 2 are the spectral energy distributions (SEDs) of several local galaxies redshifted to $z=8.23$ : the ULIRG Arp 220, the starburst M 82, the low-metallicity dwarf galaxy IZw 18, and the host galaxy of GRB 980425 $(d=40 \mathrm{Mpc}$; Michałowski et al. 2014). Our ALMA nondetection corresponds to a luminosity that is about 20 times fainter than that of Arp 220 and about 1.6 times higher than that of M 82. We also compared our limits to the galaxy templates of Rieke et al. (2009), which are based on 11 local star-forming

\footnotetext{
6 We used the following values for each spectral window and date: November 18: 2.43, 2.39, 2.29, and 2.28 Jy; November 21: 2.25, 2.24, 2.17, and 2.16 Jy; and November 30: 1.81, 1.80, 1.74, and 1.74 Jy.

7 We note the presence of a $3 \sigma$ peak at R.A. $=09^{\mathrm{h}} 55^{\mathrm{m}} 33.17$, decl. $=$ $+18^{\circ} 08^{\prime} 59^{\prime \prime} .0$ (J2000), about $2^{\prime \prime}$ away from the position of GRB 090423, but given the low signal-to-noise ratio and the substantial offset, we do not consider this potential source to be related to GRB 090423 .
} 

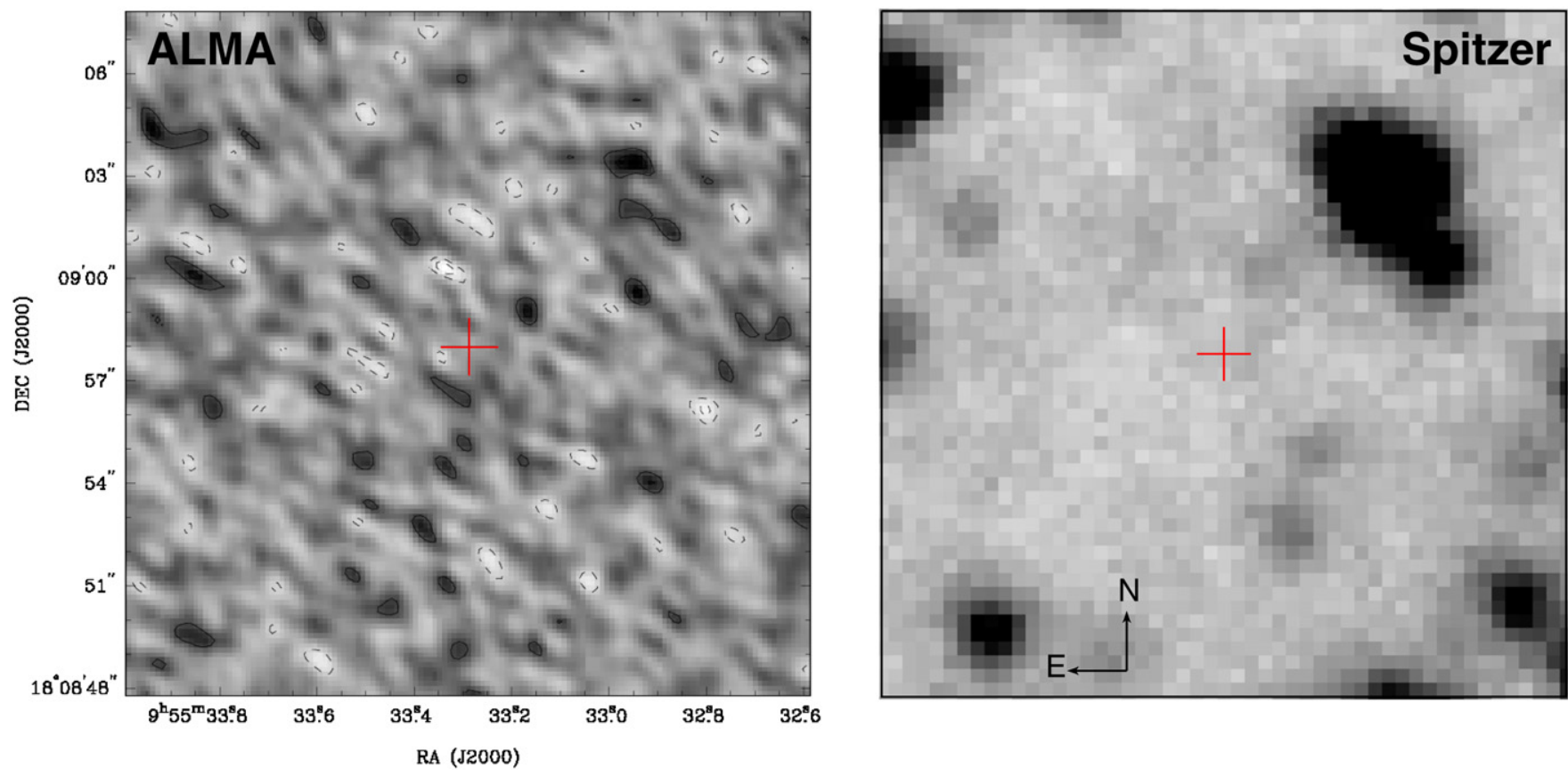

Figure 1. Left: ALMA band 6 continuum image of a $20^{\prime \prime} \times 20^{\prime \prime}$ region centered on the location of the host galaxy of GRB 090423 (cross). Contours are in steps of $1 \sigma=11 \mu \mathrm{Jy}_{\text {beam }}{ }^{-1}$ starting at $\pm 2 \sigma$ (solid: positive; dashed: negative). No millimeter emission is detected at the location of the host galaxy. Right: Spitzer/IRAC $3.6 \mu \mathrm{m}$ image of a $20^{\prime \prime} \times 20^{\prime \prime}$ region centered on the location of the host galaxy of GRB 090423 (cross). No infrared emission is detected at the location of the host galaxy.

(A color version of this figure is available in the online journal.)
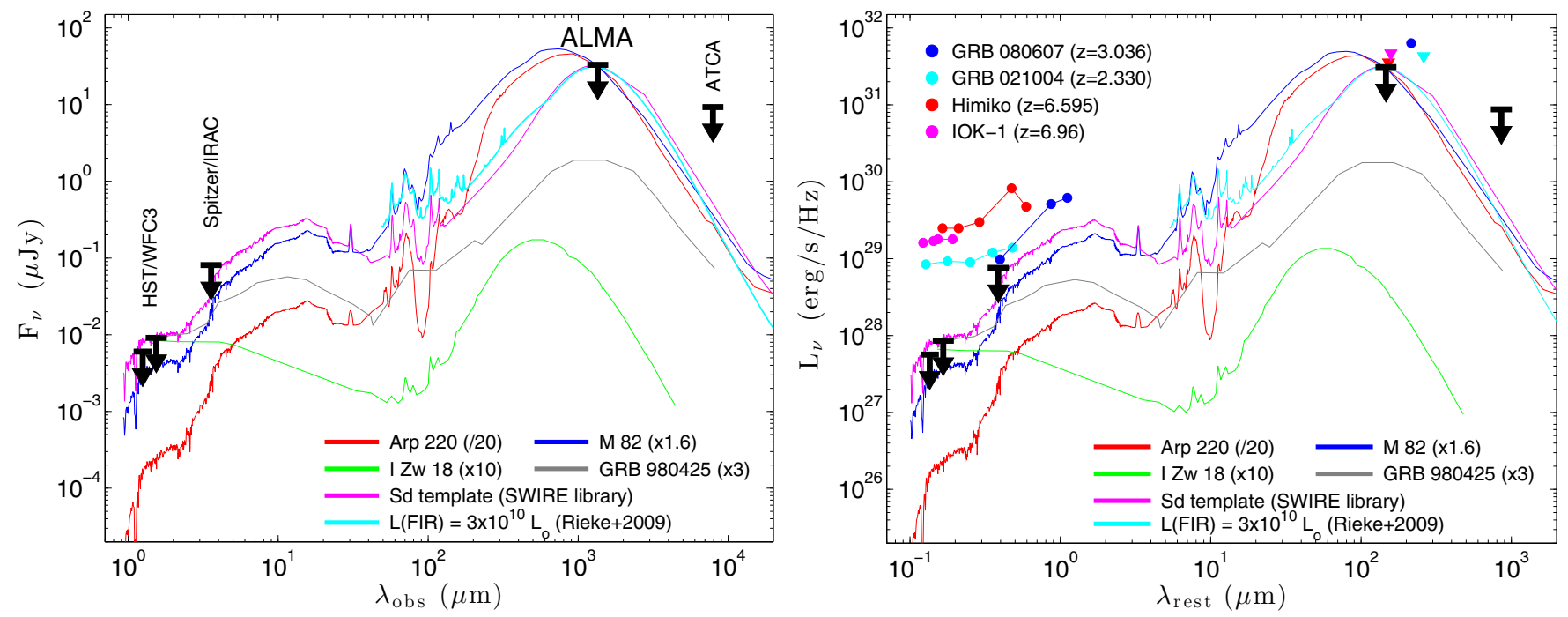

Figure 2. Left: limits on the flux density of the host galaxy of GRB 090423 in the near-IR (HST), mid-IR (Spitzer), millimeter (ALMA), and radio (ATCA). Also shown are the SEDs of the local ULIRG Arp 220 (red), the local starburst M 82 (blue), the local dwarf IZw 18 (green), the local host galaxy of GRB 980425 (gray; Michałowski et al. 2014), an Sd galaxy template (magenta), and a template for a galaxy with $L_{\mathrm{IR}}=3 \times 10^{10} L_{\odot}$ (cyan; Rieke et al. 2009), all shifted to $z=8.23$ with the exception of IZw 18 and the host of GRB 980425, which are scaled to the HST limits. The galaxy models are scaled to match the ALMA flux density limit. For the Arp 220 template, the ALMA non-detection places a stronger constraint on the SED than the HST and Spitzer limits, while for the starburst templates, the limits are comparable. For the IZw 18 and GRB 980425 host galaxy templates, the HST limits are more constraining. Right: same as the left panel, but plotting the rest-frame luminosity density and wavelength. Also shown are the ALMA observations and rest-frame UV/optical SEDs of two other GRB host galaxies (circles: detections; triangles: upper limits; GRB 080607 is a marginal 3.4 $\sigma$ detection; Wang et al. 2012), and two spectroscopically confirmed LAEs at $z \approx 6.6-7.0$ (Ouchi et al. 2013; Ota et al. 2014).

(A color version of this figure is available in the online journal.)

LIRGs and ULIRGs, and find that the ALMA non-detection corresponds to $L_{\mathrm{IR}} \lesssim 3 \times 10^{10} L_{\odot}$ (Figure 2 ).

For the Arp 220 template, the ALMA non-detection provides a much more stringent limit than the HST and Spitzer limits, which only rule out a luminosity comparable to that of Arp 220 . The ALMA constraints are also more stringent for the M 82 template, although only by about a factor of two. For an $\mathrm{Sd}$ galaxy template from the Spitzer Wide-area InfraRed Extragalactic survey library, the ALMA, HST, and Spitzer data all place comparable limits on the host SED. On the other hand, for the IZw 18 template, which has weak FIR emission, the HST limits place a much more stringent constraint than the 
Table 2

Inferred Properties of the Host Galaxy of GRB 090423

\begin{tabular}{lc}
\hline \hline Parameter & Value \\
\hline$L_{\mathrm{IR}}^{\mathrm{a}}$ & $\lesssim(2-5) \times 10^{10} L_{\odot}$ \\
$\mathrm{SFR}_{\mathrm{IR}}^{\mathrm{I}}$ & $\lesssim 3-5 M_{\odot} \mathrm{yr}^{-1}$ \\
$\mathrm{SFR}_{\mathrm{UV}}^{\mathrm{rmb}}$ & $\lesssim 1.2 M_{\odot} \mathrm{yr}^{-1}$ \\
$M_{*}^{\mathrm{c}}$ & $\lesssim(1-5) \times 10^{7} M_{\odot}$ \\
\hline
\end{tabular}

Notes.

${ }^{\text {a Assuming }} T_{\text {dust }} \approx 30-50 \mathrm{~K}$ and $\beta \approx 1.5-2$.

b Assuming $A_{V}^{\text {host }}=0 \mathrm{mag}$.

c Assuming a stellar population age of 10-100 Myr, constant star formation rate, Salpeter IMF, $Z=0.2 Z_{\odot}$, and $A_{V}^{\text {host }}=0 \mathrm{mag}$.

ALMA data by about a factor of 600 . The same is true for the GRB 980425 host galaxy template, which is about 15 times fainter than the ALMA limit when scaled to the HST limits.

Using the standard modified blackbody SED of dust emission, with a dust temperature range of $T_{\text {dust }} \approx 30-50 \mathrm{~K}$ and $\beta \approx$ 1.5-2 (e.g., Swinbank et al. 2014), the ALMA non-detection corresponds to an upper limit on the integrated IR luminosity of $L_{\mathrm{IR}}(8-1000 \mu \mathrm{m}) \lesssim(2-5) \times 10^{10} L_{\odot}$; the lower bound on the blackbody temperature is set by the cosmic microwave background temperature $T_{\mathrm{CMB}}(z=8.23) \approx 25 \mathrm{~K}$ (da Cunha et al. 2013). The IR luminosity upper limit is thus a few times lower than the scale for an LIRG and agrees well with the comparison to the Rieke et al. (2009) templates. The limit on the integrated IR luminosity corresponds to an upper bound on the obscured star formation rate (Kennicutt 1998; Calzetti et al. 2010), of ${ }^{8} \mathrm{SFR}_{\mathrm{IR}} \lesssim 3-5 M_{\odot} \mathrm{yr}^{-1}$. For comparison, the limit on the unobscured star formation rate from the HST rest-frame UV non-detection is $\mathrm{SFR}_{\mathrm{UV}} \lesssim 1.2 \mathrm{M}_{\odot} \mathrm{yr}^{-1}$.

We also place an upper bound on the host galaxy stellar mass using the HST and Spitzer upper limits. Utilizing the Bruzual \& Charlot (2003) stellar population synthesis models with a constant star formation rate, a Salpeter initial mass function (IMF), a metallicity of $0.2 Z_{\odot}$, and no dust extinction, we find that for a stellar population age of 10-100 Myr (typical of GRB host galaxies; Savaglioet al. 2009; Leibler \& Berger 2010) the limit on the host stellar mass is $M_{*} \lesssim(1-5) \times 10^{7} M_{\odot}$. The inferred host galaxy properties are summarized in Table 2.

\section{COMPARISON TO MILLIMETER OBSERVATIONS OF FIELD GALAXIES AT $z \gtrsim 4$}

To place the non-detection of the host galaxy of GRB 090423 in the context of millimeter studies of high-redshift galaxies, we summarize below previous observational efforts to detect and study distant LAEs, LBGs, and SMGs; the information is summarized in Figure 3. We first note that at present, ALMA observations of only two other galaxies with spectroscopic redshifts of $z \gtrsim 6$ have been published: the LAEs "Himiko" at $z=6.595$ (Ouchi et al. 2013; Ota et al. 2014) and IOK-1 at $z=6.96$ (Ota et al. 2014). Neither source was detected, with resulting $3 \sigma$ flux density limits of $F_{v}(259 \mathrm{GHz}) \lesssim 52 \mu \mathrm{Jy}$ and $F_{v}(232 \mathrm{GHz}) \lesssim 63 \mu \mathrm{Jy}$, respectively. The flux density and integrated IR luminosity limits for these two LAEs are shallower than those achieved in our observations; see Figure 3. However, it is important to note that Himiko and IOK-1 are significantly more luminous than the host of GRB 090423 in the rest-frame

\footnotetext{
Using the scaled SED of IZw 18, which has much weaker FIR emission relative to its optical/UV emission, leads to a limit of $\mathrm{SFR}_{\mathrm{IR}} \lesssim 100 M_{\odot} \mathrm{yr}^{-1}$.
}

UV and optical by a factor of $\gtrsim 30$ (Figure 2), with inferred unobscured star formation rates of $\approx 25-30 M_{\odot} \mathrm{yr}^{-1}$ (Ouchi et al. 2013; Ota et al. 2014). Thus, in terms of the ratio of FIR to UV luminosity, the limits for Himiko and IOK-1 are more constraining than those for the host of GRB 090423.

Beyond the ALMA observations of $z \gtrsim 6$ galaxies, there are shallower observations from other millimeter facilities. Boone et al. (2007) observed the lensed LAE HCM 6A at $z=6.56$ with the Plateau de Bure Interferometer (PdBI) and placed a limit of $L_{\mathrm{IR}} \lesssim 2.1 \times 10^{11} L_{\odot}$. Schaerer et al. (2014) also used PdBI to observe the lensed $z \approx 6.8$ LBG A1703-zD1 and the $z=7.508$ LBG 8-GND-5296, leading to limits of $L_{\mathrm{IR}} \lesssim 8.1 \times 10^{10} L_{\odot}$ (demagnified) and $L_{\mathrm{IR}} \lesssim 6.6 \times 10^{11} L_{\odot}$, respectively. GonzálezLópez et al. (2014) used the Combined Array for Research in Millimeter-wave Astronomy to place limits on two LAEs at $z=6.541$ and $z=6.554$ of $\lesssim 1.0 \times 10^{12} L_{\odot}$ and $2.1 \times 10^{12} L_{\odot}$, respectively. Riechers et al. (2013) used a wide range of facilities to study the bright SMG HFLS 3 at $z=6.337$, discovered in the Herschel Multi-tiered Extragalactic Survey, and found $L_{\mathrm{IR}} \approx 2.9 \times 10^{13} L_{\odot}$. Thus, the only galaxy at $z \gtrsim 6$ detected in the millimeter band to date is a hyperluminous infrared galaxy (Figure 3).

Expanding the redshift range down to $z=4$, only a few galaxies with spectroscopic redshifts have been detected with ALMA, all of which are bright SMGs (Figure 3). Swinbank et al. (2012) detected two SMGs in ALMA observations of the LABOCA Extended Chandra Deep Field-South Survey, with [C $\mathrm{II}] \lambda 157.74 \mu \mathrm{m}$ line emission indicating redshifts of $z=4.419$ and $z=4.444$, and resulting IR luminosities of $2 \times 10^{12} L_{\odot}$. Wagg et al. (2012) and Carilli et al. (2013) presented continuum and $\left[\mathrm{C}_{\mathrm{II}}\right]$ line emission from the SMG and two LAEs associated with the active galactic nucleus/ SMG system BRI 1202-0725 at $z=4.70$, with luminosities of $1.2 \times 10^{13} L_{\odot}(\mathrm{SMG}), \gtrsim 3.6 \times 10^{11} L_{\odot}(\mathrm{Ly} \alpha-1)$, and $1.7 \times 10^{12} L_{\odot}(\operatorname{Ly} \alpha-2)$. Hezaveh et al. (2013) detected two strongly lensed SMGs at $z=4.224$ and $z=5.656$, found in South Pole Telescope data, with intrinsic IR luminosities of about $3.8 \times 10^{12} L_{\odot}$ and $3.7 \times 10^{13} L_{\odot}$, respectively. Wiklind et al. (2014) detected an SMG at $z=4.762$, first identified in LABOCA observations of the Extended Chandra Deep Field, with $L_{\mathrm{IR}} \approx 1.3 \times 10^{13} L_{\odot}$. Riechers et al. (2014) detected the SMG AzTEC-3 at $z=5.299$ with $L_{\mathrm{IR}} \approx 1.7 \times 10^{13} L_{\odot}$, and placed a limit on an LBG in the same field at a similar redshift of $z=5.295$ of $L_{\mathrm{IR}} \lesssim 5.3 \times 10^{11} L_{\odot}$.

In addition to the ALMA observations, Cox et al. (2011) studied ID 141, a lensed galaxy at $z=4.243$ from the Herschel Astrophysical Terahertz Large Area Survey, and inferred $L_{\mathrm{IR}} \approx$ (3-8) $\times 10^{12} L_{\odot}$; the range of values accounts for the unknown lensing magnification factor. Combes et al. (2012) discovered and studied a lensed SMG at $z=5.243$ from the Herschel Lensing Survey with $L_{\mathrm{IR}} \approx 10^{13} L_{\odot}$. Walter et al. (2012) determined a redshift of $z=5.183$ for the SMG HDF 850.1 based on $\mathrm{CO}$ and $[\mathrm{CII}]$ line emission, leading to $L_{\mathrm{IR}} \approx$ $8.7 \times 10^{12} L_{\odot}$. Davies et al. (2012) used stacked MAMBO-2 observations of $z \sim 5 \mathrm{LBGs}$ to place a limit on their mean luminosity of $L_{\mathrm{IR}} \lesssim 3 \times 10^{11} L_{\odot}$, while Coppin et al. (2014) used stacked SCUBA-2 observations of $z \sim 4.8$ LBGs to find a mean luminosity of $L_{\mathrm{IR}} \sim 10^{12} L_{\odot}$ (based on a $3.8 \sigma$ detection at $850 \mu \mathrm{m})$.

Finally, two other GRB host galaxies at lower redshifts have been observed with ALMA (Wang et al. 2012). The host of GRB 021004 at $z=2.330$ was not detected, with a resulting limit of $L_{\mathrm{IR}} \lesssim 3 \times 10^{11} L_{\odot}\left(\mathrm{SFR}_{\mathrm{IR}} \lesssim 50 M_{\odot} \mathrm{yr}^{-1}\right)$. The host 


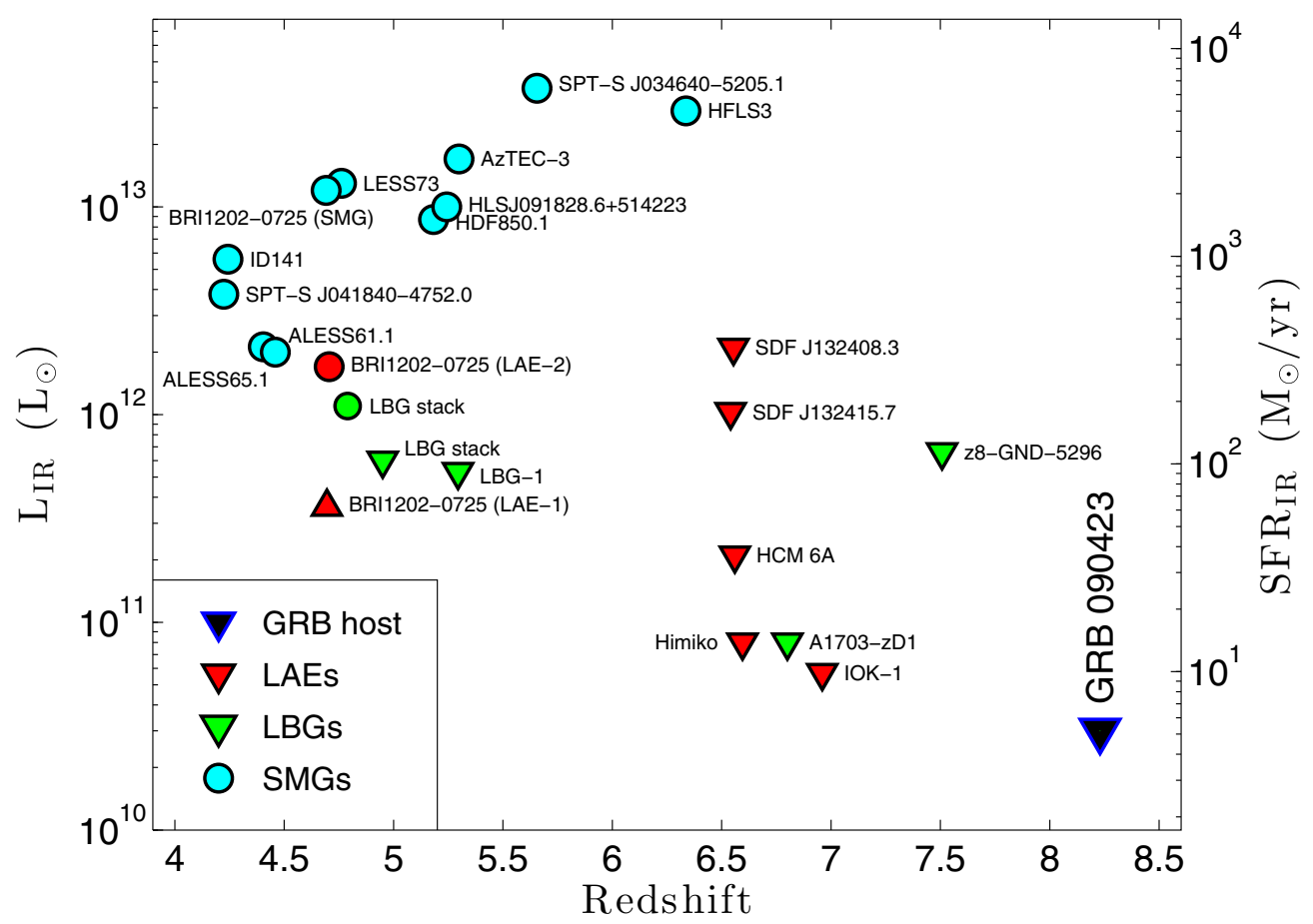

Figure 3. Integrated IR luminosities and inferred obscured star formation rates ( $\mathrm{SFR}_{\mathrm{IR}}=4.5 \times 10^{-44} L_{\mathrm{IR}} M_{\odot} \mathrm{yr}^{-1}$; Kennicutt 1998) of galaxies with spectroscopic redshifts of $z \gtrsim 4$ observed with ALMA and other millimeter telescopes (circles: detections; triangles: upper limits). The objects include SMGs (cyan), LBGs (green), LAEs (red), and the host of GRB 090423 (black). So far, mostly SMGs have been detected as individual galaxies at $z \gtrsim 4$. See Section 4 for details and references.

(A color version of this figure is available in the online journal.)

of the dusty GRB 080607 at $z=3.036$ was marginally detected $(3.4 \sigma)$ with a resulting luminosity of $(2.4-4.5) \times 10^{11} L_{\odot}$ $\left(\mathrm{SFR}_{\mathrm{IR}} \approx 40-80 M_{\odot} \mathrm{yr}^{-1}\right)$. Prior to ALMA, a few GRB host galaxies at $z \lesssim 2$ were detected in the submillimeter and radio bands (Berger et al. 2001, 2003; Frail et al. 2002; Michałowski et al. 2014; Symeonidis et al. 2014), some with inferred IR luminosities that exceed that of Arp 220. However, most GRB host galaxies were not detected at a comparable level to Arp 220 or fainter (Berger et al. 2003; Tanvir et al. 2004; Priddey et al. 2006; Perley \& Perley 2013), ruling out a dominant ULIRG host population.

Thus, in comparison to the previous studies of $z \gtrsim 4$ spectroscopically confirmed field galaxies as well as other GRB host galaxies, our ALMA observations of the host of GRB 090423 represent the deepest limit to date in terms of flux density, spectral luminosity, and integrated IR luminosity (Figure 3).

\section{CONCLUSIONS}

We present ALMA and Spitzer observations of the host galaxy of GRB 090423 at $z=8.23$, the highest-redshift spectroscopically confirmed galaxy observed with these facilities to date. The host galaxy remains undetected at rest-frame wavelength of $145 \mu \mathrm{m}$ (ALMA) and $0.39 \mu \mathrm{m}$ (Spitzer). The resulting limit on the integrated IR luminosity is $L_{\mathrm{IR}} \lesssim 3 \times 10^{10} L_{\odot}$, corresponding to $\lesssim 5 M_{\odot} \mathrm{yr}^{-1}$ of obscured star formation rate; scaling the SED of the low-metallicity dwarf galaxy IZw 18 relaxes the star formation rate upper limit inferred from the ALMA limit to $\lesssim 100 M_{\odot} \mathrm{yr}^{-1}$. In addition, based on the Spitzer and HST nondetections, we place a limit on the host galaxy stellar mass of $M_{*} \lesssim 5 \times 10^{7} M_{\odot}$ (100 Myr old stellar population with constant star formation rate). The limit on the unobscured star formation rate based on $H S T$ rest-frame UV observations is $\lesssim 1.2 M_{\odot} \mathrm{yr}^{-1}$.
We additionally compare our ALMA non-detection to millimeter observations of spectroscopically confirmed galaxies at $z \gtrsim 4$ (undertaken with ALMA and other facilities) and show that the limit on the host of GRB 090423 is the deepest to date compared to any published observations. At this redshift range, only SMGs have been convincingly detected, while individual LBGs and LAEs have so far escaped detection even with ALMA (a marginal detection of LBGs at $z \sim 4.8$ in stacked SCUBA-2 observations has been reported by Coppin et al. 2014). The only comparable limits to ours are based on ALMA observations of two LAEs at $z=6.595$ and $z=6.96$.

It is quite remarkable that we were able to reach a brightness limit of about 20 times lower than the local ULIRG Arp 220 (and comparable to the local starburst M 82) at $z=8.23$ in only $3 \mathrm{hr}$ of on-source time and with a subset of the ALMA antennas. On the other hand, if typical high-redshift galaxies have SEDs similar to the local dwarf IZw 18 or the host galaxy of GRB 980425, then much deeper ALMA observations will be required to detect their FIR emission. Still, looking forward, we anticipate that ALMA observations of GRB host galaxies will be highly desirable and productive, especially targeting spectroscopically confirmed hosts at $z \gtrsim 4$ with detailed ISM metallicity measurements, a unique sample among high-redshift galaxies.

The Berger GRB group at Harvard is supported in part by the National Science Foundation under grant AST-1107973. This paper makes use of the following ALMA data: ADS/ JAO.ALMA\#2012.1.00953.S. ALMA is a partnership of ESO (representing its member states), NSF (USA) and NINS (Japan), together with NRC (Canada) and NSC and ASIAA (Taiwan), in cooperation with the Republic of Chile. The Joint ALMA Observatory is operated by ESO, AUI/NRAO, and NAOJ. 
The National Radio Astronomy Observatory is a facility of the National Science Foundation operated under cooperative agreement by Associated Universities, Inc. This work is based in part on observations made with the Spitzer Space Telescope, which is operated by the Jet Propulsion Laboratory, California Institute of Technology under a contract with NASA.

Facilities: ALMA, Spitzer (IRAC)

\section{REFERENCES}

Berger, E., Cowie, L. L., Kulkarni, S. R., et al. 2003, ApJ, 588, 99 Berger, E., Kulkarni, S. R., \& Frail, D. A. 2001, ApJ, 560, 652

Boone, F., Schaerer, D., Pelló, R., Combes, F., \& Egami, E. 2007, A\&A, 475,513

Bouwens, R. J., Illingworth, G. D., Blakeslee, J. P., \& Franx, M. 2006, ApJ, 653,53

Bouwens, R. J., Illingworth, G. D., Oesch, P. A., et al. 2011, ApJ, 737, 90

Bruzual, G., \& Charlot, S. 2003, MNRAS, 344, 1000

Bunker, A. J., Stanway, E. R., Ellis, R. S., \& McMahon, R. G. 2004, MNRAS, 355,374

Calzetti, D., Wu, S.-Y., Hong, S., et al. 2010, ApJ, 714, 1256

Carilli, C. L., Riechers, D., Walter, F., et al. 2013, ApJ, 763, 120

Chornock, R., Berger, E., Fox, D. B., et al. 2013, ApJ, 774, 26

Chornock, R., Berger, E., Fox, D. B., et al. 2014, arXiv:1405.7400

Combes, F., Rex, M., Rawle, T. D., et al. 2012, A\&A, 538, L4

Coppin, K. E. K., Geach, J. E., Almaini, O., et al. 2014, arXiv:1407.6712

Cox, P., Krips, M., Neri, R., et al. 2011, ApJ, 740, 63

da Cunha, E., Groves, B., Walter, F., et al. 2013, ApJ, 766, 13

Davies, L. J. M., Bremer, M. N., Stanway, E. R., et al. 2012, MNRAS, 425,153

Finkelstein, S. L., Papovich, C., Dickinson, M., et al. 2013, Natur, 502, 524

Frail, D. A., Bertoldi, F., Moriarty-Schieven, G. H., et al. 2002, ApJ, 565, 829

González-López, J., Riechers, D. A., Decarli, R., et al. 2014, ApJ, 784, 99

Hezaveh, Y. D., Marrone, D. P., Fassnacht, C. D., et al. 2013, ApJ, 767, 132

Hu, E. M., Cowie, L. L., Barger, A. J., et al. 2010, ApJ, 725, 394
Iye, M., Ota, K., Kashikawa, N., et al. 2006, Natur, 443, 186

Kennicutt, R. C., Jr. 1998, ARA\&A, 36, 189

Kodaira, K., Taniguchi, Y., Kashikawa, N., et al. 2003, PASJ, 55, L17

Laskar, T., Berger, E., \& Chary, R.-R. 2011, ApJ, 739, 1

Leibler, C. N., \& Berger, E. 2010, ApJ, 725, 1202

Malhotra, S., Rhoads, J. E., Pirzkal, N., et al. 2005, ApJ, 626, 666

McLure, R. J., Dunlop, J. S., de Ravel, L., et al. 2011, MNRAS, 418, 2074

Michałowski, M. J., Hunt, L. K., Palazzi, E., et al. 2014, A\&A, 562, A70

Ono, Y., Ouchi, M., Mobasher, B., et al. 2012, ApJ, 744, 83

Ota, K., Walter, F., Ohta, K., et al. 2014, ApJ, 792, 34

Ouchi, M., Ellis, R., Ono, Y., et al. 2013, ApJ, 778, 102

Ouchi, M., Mobasher, B., Shimasaku, K., et al. 2009, ApJ, 706, 1136

Ouchi, M., Shimasaku, K., Furusawa, H., et al. 2010, ApJ, 723, 869

Perley, D. A., \& Perley, R. A. 2013, ApJ, 778, 172

Priddey, R. S., Tanvir, N. R., Levan, A. J., et al. 2006, MNRAS, 369, 1189

Riechers, D. A., Bradford, C. M., Clements, D. L., et al. 2013, Natur, 496, 329

Riechers, D. A., Carilli, C. L., Capak, P. L., et al. 2014, arXiv:1404.7159

Rieke, G. H., Alonso-Herrero, A., Weiner, B. J., et al. 2009, ApJ, 692, 556

Salvaterra, R., Della Valle, M., Campana, S., et al. 2009, Natur, 461, 1258

Savaglio, S., Glazebrook, K., \& Le Borgne, D. 2009, ApJ, 691, 182

Schaerer, D., Boone, F., Zamojski, M., et al. 2014, arXiv:1407.5793

Shibuya, T., Kashikawa, N., Ota, K., et al. 2012, ApJ, 752, 114

Stanway, E. R., Bremer, M. N., Tanvir, N. R., Levan, A. J., \& Davies, L. J. M. 2011, MNRAS, 410, 1496

Stanway, E. R., Bunker, A. J., \& McMahon, R. G. 2003, MNRAS, 342, 439

Swinbank, A. M., Karim, A., Smail, I., et al. 2012, MNRAS, 427, 1066

Swinbank, A. M., Simpson, J. M., Smail, I., et al. 2014, MNRAS, 438, 1267

Symeonidis, M., Oates, S. R., de Pasquale, M., et al. 2014, MNRAS, 443, L124

Tanvir, N. R., Barnard, V. E., Blain, A. W., et al. 2004, MNRAS, 352, 1073

Tanvir, N. R., Fox, D. B., Levan, A. J., et al. 2009, Natur, 461, 1254

Tanvir, N. R., Levan, A. J., Fruchter, A. S., et al. 2012, ApJ, 754, 46

Totani, T., Kawai, N., Kosugi, G., et al. 2006, PASJ, 58, 485

Vanzella, E., Pentericci, L., Fontana, A., et al. 2011, ApJL, 730, L35

Wagg, J., Wiklind, T., Carilli, C. L., et al. 2012, ApJL, 752, L30

Walter, F., Decarli, R., Carilli, C., et al. 2012, Natur, 486, 233

Wang, W.-H., Chen, H.-W., \& Huang, K.-Y. 2012, ApJL, 761, L32

Wiklind, T., Conselice, C. J., Dahlen, T., et al. 2014, ApJ, 785, 111 